\title{
A Disposable Inkjet Head Driven by Pulsed Laser
}

\author{
Toshihiko OOIE, ${ }^{1,5}$ Masato TANAKA,,${ }^{1,5}$ Tomonori NAKAHARA, ${ }^{1}$ Yuji YAMACHOSHI, ${ }^{1}$ \\ Hidetoshi MIYASHITA, ${ }^{1}$ Shinji HYODO, ${ }^{2}$ Yasuo SHINOHARA, ${ }^{3}$ and Yoshinobu BABA ${ }^{1,4}$ \\ ${ }^{1}$ AIST, Health Technology Research Center, Japan, 2217-14 Hayashi-cho, Takamatsu, Kagawa 761-0395 \\ ${ }^{2}$ Toyo Manufacturing Co., Ltd., 343-3 Kitanoda, Tohon, Ehime 791-0215 \\ ${ }^{3}$ Institute for Genome Research, The University of Tokushima, 3-8-15 Kuramoto-cho, Tokushima 770-8503 \\ ${ }^{4}$ Graduate School of Engineering, Nagoya University, Furo-cho, Chikusa-ku, Nagoya 464-8603 \\ ${ }^{5}$ Graduate School of Advanced Science and Technology, The University of Tokushima, \\ 2-1 Minamijosanjima, Tokushima 770-8506
}

(Received January 29, 2008)

\begin{abstract}
This article describes the development and evaluation of a disposable inkjet head driven by a pulsed laser. Since it has a very simple structure compared to other conventional inkjet heads, it is suitable for disposable devices such as various nano-biodevices for Point of Care Testing (POCT). The driving unit in the inkjet head is merely a piece of metal foil without any electric signal or power line. A laser and optics external to the chip provide the energy to drive the head. Explosive vapor bubbles formed on the metal foil in a microchannel during laser irradiation successfully ejected diluted ink and biopolymer samples in water solution, respectively, from the channel as small droplets through the ejection port. The flight speed of the ejected droplets reached $7.2 \mathrm{~m} / \mathrm{s}$. The biopolymer samples before and after ejection indicated similar mass spectra, as measured by surface-enhanced laser desorption ionization time of flight mass spectroscopy (SELDI-TOF MS).
\end{abstract}

Key Words: Nano-biodevice, Inkjet, Microfluidic, Biopolymer, Protein, Pulsed laser, Fiber laser, PMMA, Bubble, Droplet

\section{Introduction}

In typical micro Total Analysis Systems (micro TAS) ${ }^{1-3)}$, most functions involving several analyzers are integrated on a single chip. However, their driving unit, whether piezoelectric actuators ${ }^{4)}$, electrochemical actuators ${ }^{5)}$, micro valves ${ }^{6)}$ or micro heaters ${ }^{7)}$ are not suitable for plastic-based disposable chips for Point of Care Testing (POCT) ${ }^{8)}$ in the medical field because of the cost involved.

New units including a micro flow switch using thermal gelation of methyl cellulose ${ }^{9}$, an optical switching valve using the change of surface property ${ }^{10)}$ and a pump using a laser manipulation technique ${ }^{11)}$ were developed to reduce the number of parts on the chip. By using an external light source as the driving force, the signal line between the chip and the external controller was successfully eliminated Furthermore, these units have the capability of multipoint control by a single light source with a scanning optic.

Laser driven inkjet units have merits similar to these devices. J. Deoksuk et. al. experimentally analyzed the bubble dynamics of ink, the absorption coefficient of which was sufficient for direct heating, induced by laser irradiation ${ }^{12)}$. Their results show that the bubble generated in the ink by a laser pulse is similar to that produced by an electric thin film heater.

Indirect laser heating via metal film was applied for the phosphor layer deposition to a plasma display panel (PDP) by J. H. Lee et. al. ${ }^{13)}$. However, their process is a transfer rather than an inkjet, since they used a shallow, hemispherical, blind hole without any supply line to refill liquid.

Transfer techniques involving laser-induced forward transfer (LIFT) ${ }^{14)}$ have been studied for biological applications ${ }^{15-19)}$ also. Various biological materials including proteins, DNA and cells have been transferred without significant damage.

We have developed a disposable inkjet head driven by pulsed laser. It is a drop-on-demand head, applicable to laser-transparent liquid.

\section{Experimental}

Figure 1 shows a schematic diagram of our experimental inkjet system. The system contains two major units; an inkjet head itself and a laser.

The inkjet head consists of three parts; a base plate with microgroove, a piece of thin metal foil as laser absorber and a cover film. To create a single, experimental nozzle head, a commercially available injection-molded electrophoresis chip made of PMMA (IC-1000, Hitachi High-Technologies) was used as the base plate. The groove width was $120 \mu \mathrm{m}$ at the surface reducing to $50 \mu \mathrm{m}$ at the bottom. The depth of the groove was $30 \mu \mathrm{m}$.

Two kinds of absorbers were made from commercially available materials, one in titanium and the other in nickel, with thicknesses of $2 \mu \mathrm{m}$ and $2.5 \mu \mathrm{m}$ respectively. The absorber was positioned on the groove before the groove was sealed by 


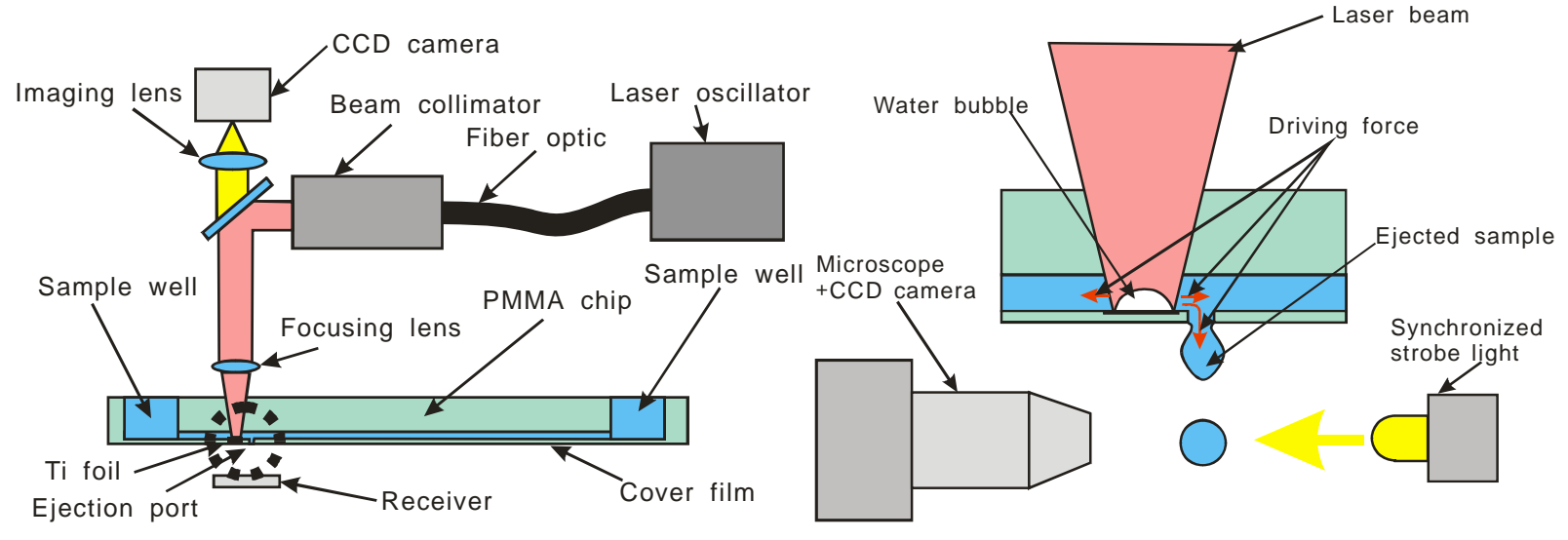

Fig. 1 Schematic diagram of experimental inkjet system and droplet imaging.

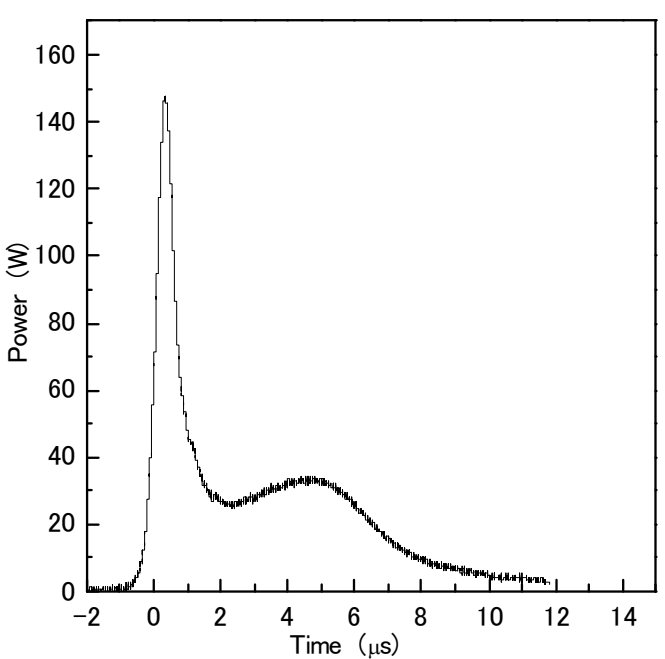

Fig. 2 Typical pulse waveform of the fiber laser used as inkjet driver.

a thin polymethylmethacrylate (PMMA) film coated with acrylate adhesive. The thickness of the film itself and that of the adhesive layer were $30 \mu \mathrm{m}$ and $10 \mu \mathrm{m}$, respectively. The film was originally developed as the cover film for electrophoresis chips under collaboration with Toyo Ink Mfg. Co., Ltd.

A femtosecond laser, the repetition rate, pulse width and wavelength of which were $1 \mathrm{kHz}, 200 \mathrm{fs}$ and $780 \mathrm{~nm}$, respectively, and a microscope objective lens, with Numerical Aperture (NA) and focal length (f) of 0.55 and 4mm, respectively, was used to cut thin metal foils into small pieces suitable for laser absorbers. It was also used to drill the ejection port on the PMMA film.

A fiber laser (wavelength 1060nm, IPG Photonics, YLD-40) was used to drive inkjet head. Its typical pulse waveform is shown in Fig.2. The pulse consisted of two parts; an initial high, narrow peak with full width at half maximum which had a constant value of approximately $1 \mu$ s, and a tail that depended on the pulse width. The peak level of the former was 4 times higher than the average level of the latter. The laser beam was focused by a lens, the focal length of which was $40 \mathrm{~mm}$, onto the absorber. The focus was set at $300 \mu \mathrm{m}$ below the absorber

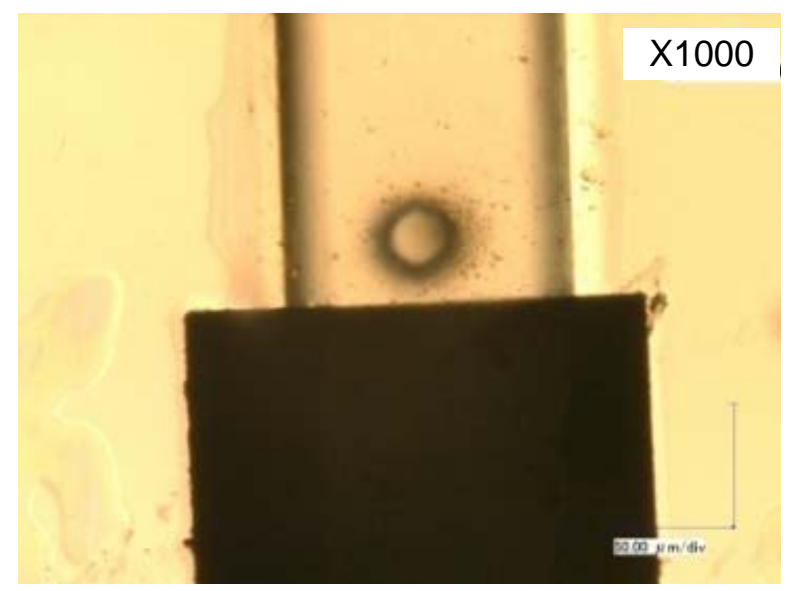

Fig. 3 Photograph of an inkjet head that consists of a commercially available electrophoresis chip, an absorber and a PMMA cover film with ejection port.

surface creating a beam diameter of $75 \mu \mathrm{m}$. A CCD camera equipped with synchronized strobe light unit, the minimum flashing time of which was less than $1 \mu$ s, was used to take images of ejected droplets. A white graph paper at a distance of $5 \mathrm{~mm}$ from the ejection port was used to evaluate the directional accuracy of the inkjet system.

Diluted red ink and four protein samples; cytochrome-c in $0.1 \mathrm{mg} / \mathrm{ml}$ of water and in $1 \mathrm{mg} / \mathrm{ml}$ of water, and bovine serum albumin (BSA) in $0.1 \mathrm{mg} / \mathrm{ml}$ of water and in $1 \mathrm{mg} / \mathrm{ml}$ of water, respectively, were used for the experiments. Protein samples ejected to a protein chip array for surface-enhanced laser desorption ionization-time of flight mass spectrometry (SELDI-TOF MS) were analyzed to compare their molecule size after ejection with that of the originals.

\section{Results and Discussion}

The experimental inkjet head shown in Fig. 3 was composed of an absorber, an ejection port and a commercially available electrophoresis chip. In this head, the absorber is a piece of nickel foil $150 \mu \mathrm{m}$ square and $2.5 \mu \mathrm{m}$ thick.

The images of droplets at various time delays after the laser 


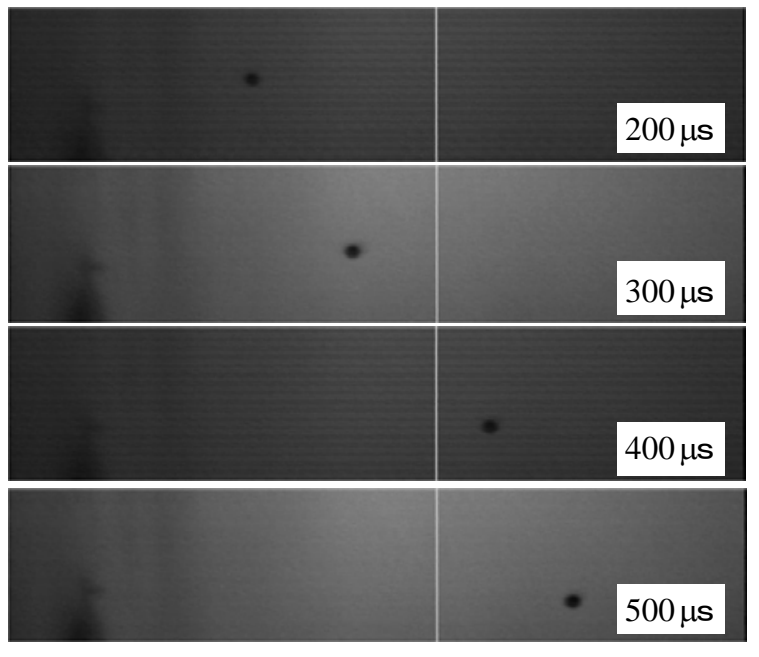

Fig. 4 Droplet images at various time delays after ejection.

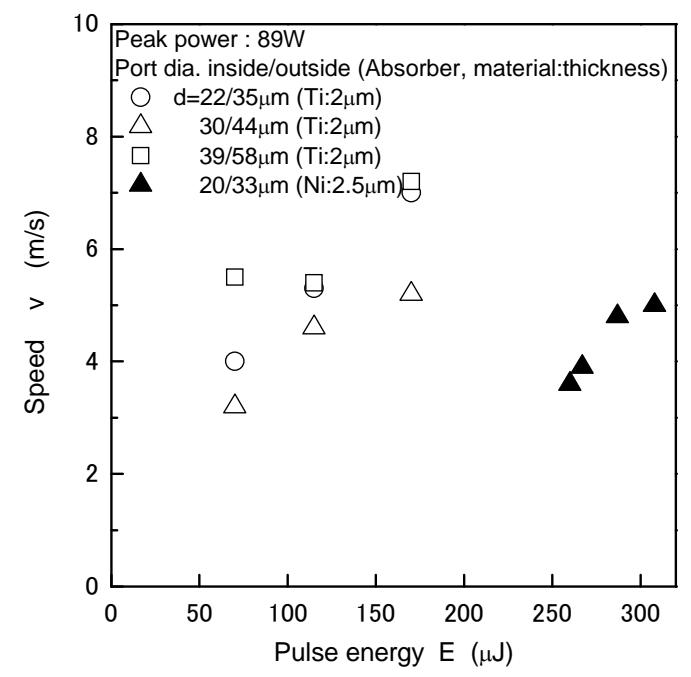

Fig. 6 Relationship between the flight speed of ejected droplets and laser pulse energy.

pulse began to rise are shown in Fig. 4. According to a series of images taken at various pulse energies and port diameters, the diameters of the droplets depended largely on the diameter of the ejection port and much less on the pulse energy of the laser. As shown in Fig. 5, the distance from the ejection port to the droplet is proportional to the delay time up to $400 \mu \mathrm{s}$, showing that the flight speed of the droplet is constant in that region.

The speed of ejected droplets depended on the pulse energy of the laser, as shown in Fig. 6. The estimated flight speed of the droplets when using the titanium absorber was in the range of 3 to $7.2 \mathrm{~m} / \mathrm{s}$ with pulse energy of up to $170 \mu \mathrm{J}$. As shown in the same figure, the nickel absorber required triple the pulse energy of titanium. These two materials have quite different thermal conductivity and reflectivity ${ }^{20-21)}$. Further, the heat capacity of the nickel absorber is two times larger than that of the titanium absorber, owing to the differences in their specific gravity, specific heat and thickness. If each absorber was thermally isolated, their average temperature increases for $\mathrm{v}=4 \mathrm{~m} / \mathrm{s}$ would be both approximately 370 degrees. The

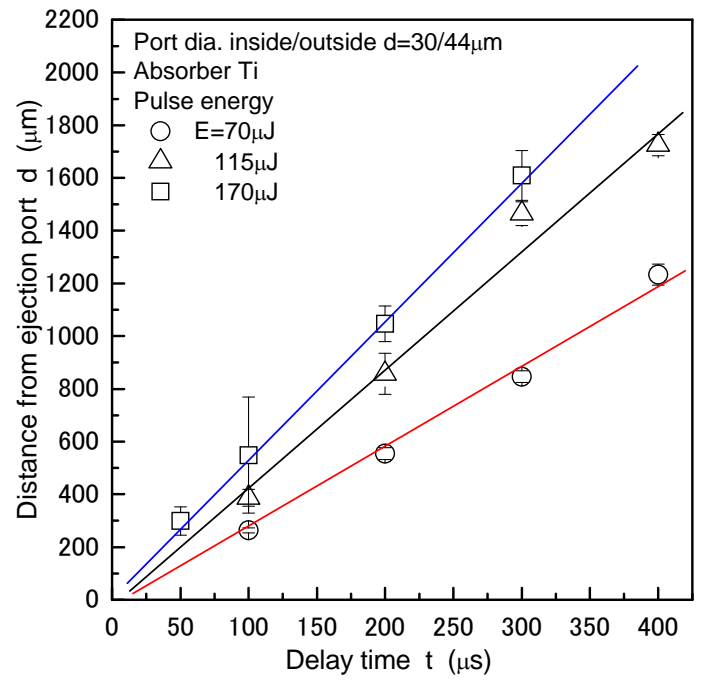

Fig. 5 Flight distance of droplets from ejection port at various time delays after ejection.

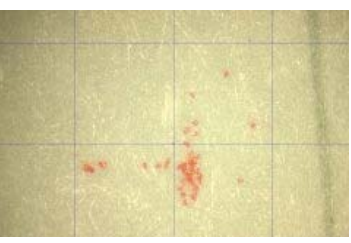

(a) $\mathrm{E}=120 \mu \mathrm{J}, 60$ pulses

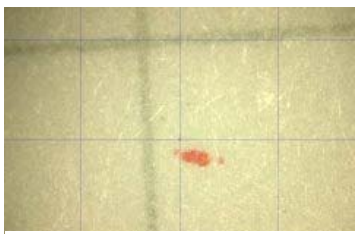

(c) $E=190 \mu \mathrm{J}, 30$ pulses

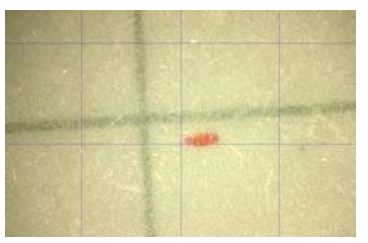

(b) $E=150 \mu \mathrm{J}, 30$ pulses

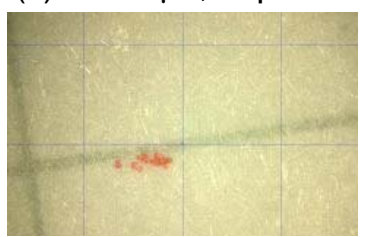

(d) $E=230 \mu \mathrm{J}, 30$ pulses
Fig. 7 Ejected ink patterns at a distance of $5 \mathrm{~mm}$ from the ejection port.

maximum energy efficiencies of the experimental head with titanium absorber and that with nickel absorber were approximately $5.7 \times 10^{-5}$ and $2.3 \times 10^{-6}$, respectively. These values are still low compared to commercially available thermal inkjet heads driven by electric heaters, whose heat efficiency is $1-4 \times 10^{-4}$ 22). The heat efficiency of the head can be improved while retaining its simple structure, by reducing the heat capacity of the absorber.

Figure 7 shows the ejected patterns of diluted red ink at a distance of $5 \mathrm{~mm}$ from the ejection port, when using a titanium absorber. The frequency and pulse width of the laser were constant at $1 \mathrm{~Hz}$ and $2 \mu \mathrm{s}$, respectively. Although the dispersion of the droplets was rather large at a pulse energy of $120 \mu \mathrm{J}$, it decreased with increasing pulse energy.

Figure 8 shows the SELDI-TOM MS signals of the BSA samples before and after ejection. There is no significant difference between the signal of the ejected sample and that from the original sample. Similar results were obtained for cytochrome-c. In addition, the signal level of SELDI-TOF MS was proportional to the number of laser pulses, which is equal 


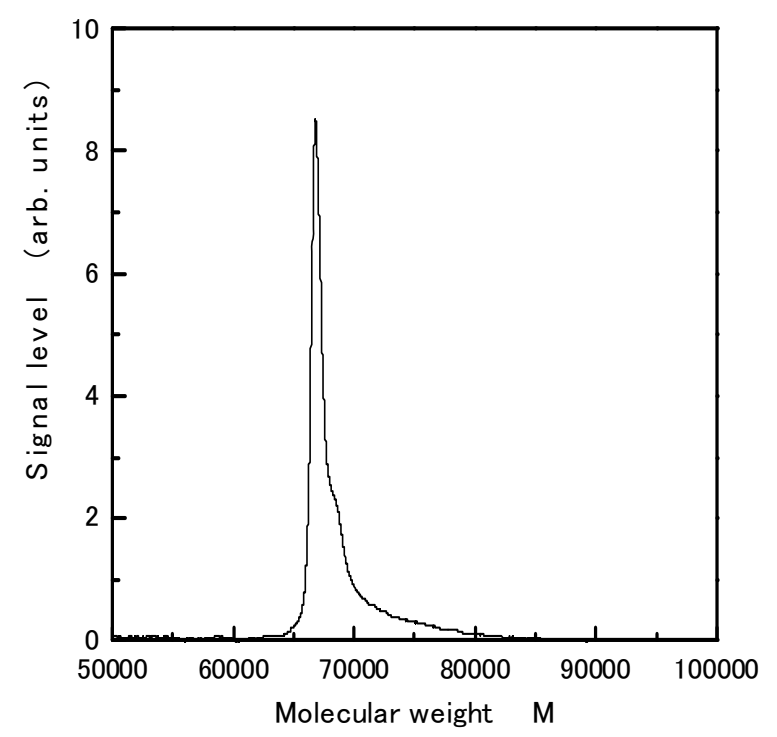

(a) MS signal of BSA before ejection.

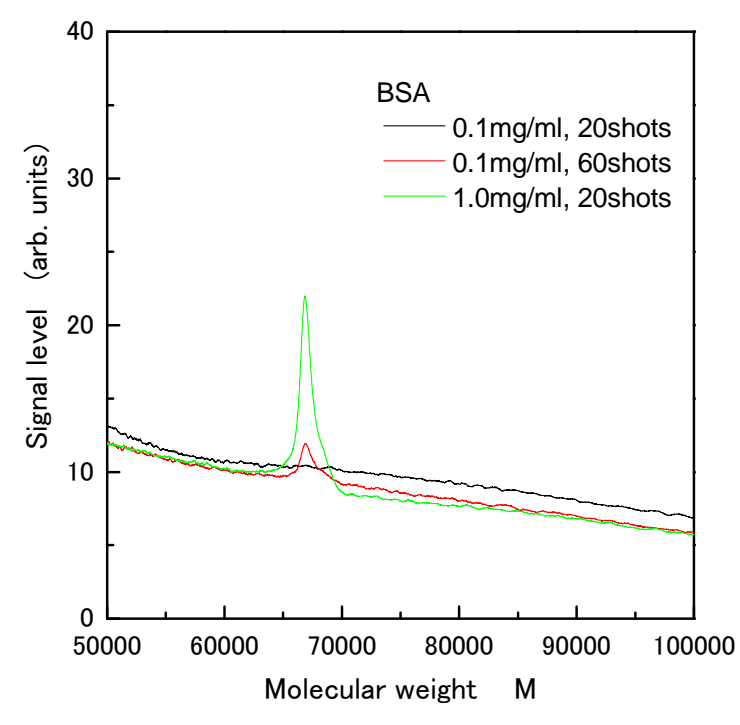

(b) MS signal of BSA after ejection.

Fig. 8 SELDI-TOF MS signals of BSA sample before and after ejection.

to the number of droplets, demonstrating that the inkjet head is applicable to the quantitative handling of biopolymers. The volume of a single drop depended on the size of the exit port.

\section{Conclusion}

A disposable inkjet head driven by a pulsed laser was constructed experimentally. An explosive bubble formed by laser irradiation successfully ejected liquid samples from the channel on a PMMA chip via an ejection port. The speed of the ejected droplets depended on the laser pulse energy. Ejected protein samples had the same SELDI-TOF MS signal as the reference samples before ejection. Our inkjet head has a very simple structure and, hence, it is applicable even to disposable chips.

\section{References}

1) R. Koyama, N. Okayama, K. Shimoide, M. Kitaoka and T. Kitamori: Proc. 9th Int. Conf. on Miniaturized Systems for Chemistry and Life Sciences, Boston (USA), (2005) p.1324.

2) N. Goedecke, B. McKenna, S. El-Difrawy, J. Aborn, E. Gismondi and D. J. Ehrlich: Proc. 9th Int. Conf. on Miniaturized Systems for Chemistry and Life Sciences, Boston (USA), (2005) P.1361.

3) P. A. Auroux, Y. Koc, A. J. de Mello, A. Manz and P. J. R. Day: Lab on a Chip, 4 (2004) 534

4) T. Aono, A. Koide, R. Miyake and T. Kitamori: Proc. 9th Int. Conf. on Miniaturized Systems for Chemistry and Life Sciences, Boston (USA), (2005) p.1186.

5) S. Böhm, B. Timmer, W. Olthuis, P. Bergveld: J. Micromechanics and Microengineering, 4 (2000) 498

6) K.Hosokawa and R. Maeda: J. Micromech. Microeng., 10 (2000) 415.

7) S. U. Son, D. Kim and S. S. Lee: Proc. 9th Int. Conf. on Miniaturized Systems for Chemistry and Life Sciences, Boston
(USA), (2005) p.1084.

8) E. Eteshola, D. Leckband: Sensors and Actuators B-Chemical 72 (2001) 129.

9) Y. Shirasaki, H. Makazu, K. Tashiro, S. Ikeda, T. Sekiguchi, S. Shoji, S. Tsukita and T. Funatsu: Proc. 6th Int. Conf. on Miniaturized Systems for Chemistry and Life Sciences, Nara (Japan), (2002) p.925.

10) H. Nagai, T. Irie and S. Wakida: Proc. 9th Int. Conf. on Miniaturized Systems for Chemistry and Life Sciences, Boston (USA), (2005) p.572.

11) S. Maruo and H. Inoue: Proc. 9th Int. Conf. on Miniaturized Systems for Chemistry and Life Sciences, Boston (USA), (2005) p.590.

12) J. Deoksuk, J. Hong, K. Dongsik, C. Sung-Hoon: J. Imaging Sci. Technol., 50 (2006) 173.

13) J. H. Lee, C. D. Yoo and YS. Kim: J. Micromech Microeng, 17 (2007) 258.

14) D. A. Willis and V. Grosu: Appl. Phys. Lett. 86 (2005) 244103.

15) P. Serra, M. Colina and J. M. Fernandez-Pradas: Appl. Phys. Lett. 85 (2004) 1639.

16) B. R. Ringeisen, H. Kim, J. A. Barron, D. B. Krizman, D. B. Chrisey, S. Jackman, R. Y. C. Auyeung, B. J. Spargo: Tissue Engineering, 10 (2004) 483.

17) M. Colina, M. Duocastella, J. M. Fernandez-Pradas, P. Serra and J. L. Morenza: J. Appl. Phys. 99 (2006) 084909.

18) P. Serra, J. M. Fernandez-Pradas, M. Colina, M. Duocastella, J. Dominguez and J. L. Morenza: J. Laser Micro/Nanoengineering, 1 (2006) 236.

19) M. Duocastella, J. M. Fernandez-Pradas, P. Serra, J. L. Morenza: Proc. LPM2007, Viena (Austria), (2007), on line.

20) E. Taralli, C. Portesi, C. Ricciardi, E. Tresso, M. Rajteri: Nuc. Inst. and Methods in Phys. Res. A, 559 (2006) 757.

21) W. M. Steen: Laser Material Processing (Springer-Verlag, London, 1991) p.47.

22) M. Fujii, T. Hamazaki and K. Ikeda: J. of Imaging Sci. and Technol., 43 (1999) 332. 